\title{
Editorial Comment: Techniques - Ultrasound-guided percutaneous nephrolithotomy: How we do it
}

\author{
Beiko D ${ }^{1}$, Razvi H ${ }^{2}$, Bhojani N ${ }^{3}$, Bjazevic J ${ }^{2}$, Bayne DB ${ }^{4}$, Tzou DT ${ }^{5}$, et al. \\ ${ }^{1}$ Department of Urology, Queen's University, Kingston, ON, Canada; ${ }^{2}$ Division of Urology, Department of Surgery, Western \\ University, London, ON, Canada; ${ }^{3}$ Division of Urology, Université de Montréal, Montreal, OC, Canada; ${ }^{4}$ Department of \\ Urology, University of California at San Francisco, San Francisco, CA, United States; ${ }^{5}$ Division of Urology, Department of \\ Surgery, University of Arizona College of Medicine, Tucson, AZ, United States
}

Can Urol Assoc J. 2019 Sep 27. [Epub ahead of print]

DOI: 10.5489/cuaj.6076 | ACCESS: 10.5489/cuaj.6076

\begin{abstract}
Alexandre Danilovic ${ }^{1}$
${ }^{1}$ Serviço de Urologia, Hospital das Clínicas da Faculdade de Medicina da USP - HCFMUSP, São Paulo, SP, Brasil
\end{abstract}

\section{COMMENT}

Ultrasound-guided percutaneous nephrolithotomy (US-PNL) has gained popularity in many parts of the world in the past few years (1). It may be used in combination with fluoroscopy to reduce ionizing radiation exposure or replacing fluoroscopy to completely eliminate exposure to radiation during PNL (2). However, this technique can be challenging to learn.

This article provides some interesting key points for those who would like to start a US-PNL program. Despite other authors claim no different results for obese vs. non-obese patients (3), according to the authors, the ideal candidate to start a learning curve of US-PNL is a healthy non-obese patient whose imaging demonstrates a non-staghorn calculus and at least moderate hydronephrosis. The authors described in details eight steps for a successful US-PNL.

There are many advantages of the use of ultrasound in PNL and this technique should be encouraged. However, some anatomic details of the collecting system are missed by ultrasound. Therefore, it is recommended to progressively convert fluoroscopy to ultrasound guidance step-by-step as one gains experience. More important than completely eliminate exposure to radiation is to keep patient safe from injuries due to suboptimal image guidance. 


\section{CONFLICT OF INTEREST}

None declared.

\section{REFERENCES}

1. TLi J, Xiao B, Hu W, Yang B, Chen L, Hu H, et al. Complication and safety of ultrasound guided percutaneous nephrolithotomy in 8,025 cases in China. Chin Med J (Engl). 2014;127:4184-9.

2. Zhu W, Li J, Yuan J, Liu Y, Wan SP, Liu G, et al. A prospective and randomised trial comparing fluoroscopic, total ultrasonographic, and combined guidance for renal access in mini-percutaneous nephrolithotomy. BJU Int. 2017;119:612-8.

Alexandre Danilovic, MD

3. Jin W, Song Y, Fei X. Does Body Mass Index Impact the Outcomes of Ultrasound-Guided Percutaneous Nephrolithotomy? Urol Int 2019;103:149-55.

ARTICLE INFO

Serviço de Urologia, Hospital das Clínicas da Faculdade de Medicina da USP - HCFMUSP, São Paulo, SP, Brasil

E-mail: alexandre.danilovic@gmail.com

Alexandre Danilovic https://orcid.org/0000-0002-6963-6117

Int Braz J Urol. 2020; 46: 845-6 